\section{RECENT STUDIES IN GRAVITATION.'}

THE studies in gravitation which I am to describe to you this evening will perhaps fall into better order if I rapidly run over the well beaten track which leads to those studies, the track first laid down by Newton, based on astronomical observations, and only made firmer and broader by every later observation.

I may remind you, then, that the motion of the planets round the sun in ellipses, each marking out the area of its orbit at a constant rate, and each having a year proportional to the square root of the cube of its mean distance from the sun, implies that there is a force on each planet exactly proportional to its mass, directed towards, and inversely as the square of its distance from the sun. The lines of force radiate out from the sun on all sides equally, and always grasp any matter with a force proportional to its mass, whatever planet that matter belongs to.

If we assume that action and reaction are equal and opposite then each planet acts on the sun with a force proportional to its own mass; and if, further, we suppose that these forces are merely the sum totals of the forces due to every particle of matter in the bodies acting, we are led straight to the law of gravitation, that the force between two masses $M_{1} M_{2}$ is always proportional to the product of the masses divided by the square of the distance $r$ between them, or is equal to

$$
\mathrm{G} \times \mathrm{M}_{1} \times \mathrm{M}_{2}
$$

and the constant multiplier $\mathrm{G}$ is the constant of gravitation.

Since the force is always proportional to the mass acted on, and produces the same change of velocity whatever that mass may be, the change of velocity tells us nothing about the mass in which it takes place, but only about the mass which is pulling. If, however, we compare the accelerations due to different pulling bociies, as for instance that of the sun pulling the earth with that of the earth pulling the moon, or if we compare changes in motion due to the different planets pulling each other, then we can compare their masses and weigh them, one against another and each against the sun. But in this weighing our standard weight is not the pound or kilogramme of terrestrial weighings, but the mass of the sun.

For instance, from the fact that a body at the earth's surface, 4000 miles, on the average, from the mass of the earth, falls with a velocity increasing by $32 \mathrm{ft}$. $/ \mathrm{sec} .{ }^{2}$, while the earth falls towards the sun, 92 million miles away, with a velocity increasing by about $\frac{1}{3}$ inch $/ \mathrm{sec}^{2}{ }^{2}$, we can at once show that the mass of the sun is 300,000 times that of the earth. In other words, astronomical observation gives us only the acceleration, the product of $\mathrm{G} \times$ mass acting, but does not tell us the value of $\mathrm{G}$ nor of the mass acting, in terms of our terrestrial standards.

To weigh the sun, the planets, or the earth, in pounds or kilogrammes, or to find $\mathrm{G}$, we must descend from the heavenly bodies to earthly matter and either compare the pull of a weighable mass on some body with the pull of the earth on it, or else choose two weighable masses and find the pull between them.

All this was clearly seen by Newton, and was set forth in his "System of the World" (third edition, p. 4I).

He saw that a mountain mass might be used, and weighed against the earth by finding how much it deflected the plumb line at its base. The density of the mountain could be found from specimens of the rocks composing it, and the distance of its parts from the plumb line by a survey. The defiection of the vertical would then give the mass of the earth.

Newton also considered the possibility of measuring the attraction between two weighable masses, and calculated how Iong it would take a sphere a foot in diameter, of the earth's mean density, to draw another equal sphere, with their surfaces separated by $\frac{1}{4}$-inch, through that $\frac{1}{2}$-inch. But he made a very great mistake in his arithmetic, for while his result gave about one month, the actual time would only be about $5 \frac{1}{2}$ minutes. Had his value been right, gravitational experiments would have been beyond the power of even Prof. Boys. Some doubt has been thrown on Newton's authorship of this mistake, but I confess that there is something not altogether unpleasing even in the mistake of a Newton. His faulty

1 A discourse delivered at the Royal Institution of Great Britain on Friday, February 23, by Prof. John H. Poynting, F.R.S. arithmetic showed that there was one quality which he shared with the rest of mankind.

Not long after Newton's death the mountain experiment was actually tried, and in two ways. The honour of making these first experiments on gravitation belongs to Bouguer, whose splendid work in thus breaking new ground does not appear to me to have received the credit due to it.

One of his plans consisted in measuring the deflection of the plumb line due to Chimborazo, one of the Andes peaks, by finding the distance of a star on the meridian from the zenith, first at a station on the south side of the mountain, where the vertical was deflected, and then at a station to the west, where the mountain attraction was nearly inconsiderable, so that the actual nearly coincided with the geographical vertical. The difference in zenith distances gave the mountain deflection. It is not surprising that, working in snowstorms at one station, and in sandstorms at the other, Bouguer obtained a very incorrect result. But at least he showed the possibility of such work, and since his time many experiments have been carried out on his lines under more favourable conditions. Now, however, I think it is generally recognised that the difficulty of estimating the mass of a mountain from mere surface chips is insurmountable, and it is admitted that the experiment should be turned the other way about and regarded as an attempt to measure the mass of the mountains from the density of the earth known by other experiments.

These other experiments are on the line indicated by Newton in his calculations of the attraction of two spheres. The first was carried out by Cavendish.

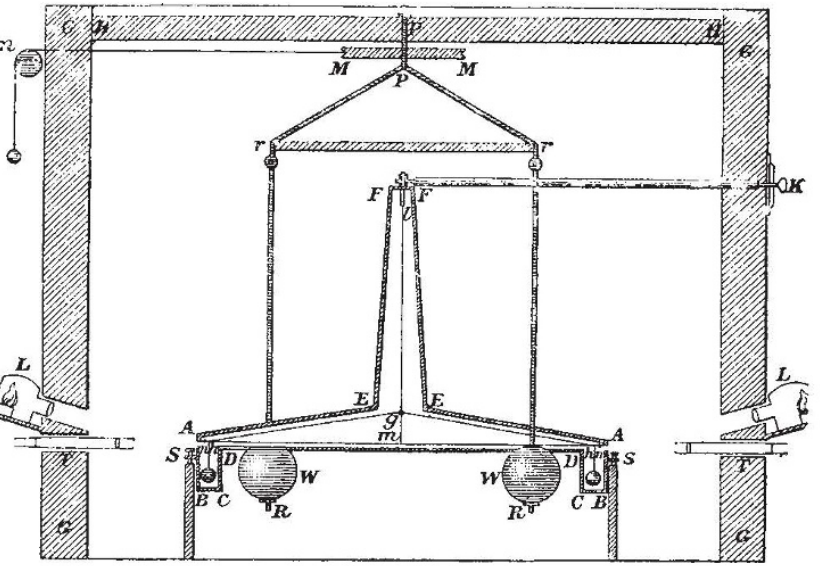

F'IG. I.—Cavendish's apparatus.

In the apparatus (Fig. I) he used two lead balls, B B, each $2^{\prime \prime}$ in diameter. These were hung at the end of a horizontal rod $6^{\prime}$ long, the torsion rod, and this was hung up by a long wire from its middle point. Two large attracting spheres of lead, $w$ w, each $\mathrm{I} 2^{\prime \prime}$ in diameter, were brought close to the balls on opposite sides so that their attractions on the balls conspired to twist the torsion rod round the same way, and the angle of twist was measured. The force could be reckoned in terms of this angle by setting the rod vibrating to and fro and finding the time of vibration, and the force came out to less than $\mathrm{I} / 3000$ of $a$ grain. Knowing $M_{3} M_{2}$ and $r$ the distance between them and the force G $M_{1} M_{2} / r^{2}$, of course Cavendish's result gives $G$, or knowing the attraction of a big sphere on a ball, and knowing the attraction of the earth on the same ball, that is, its weight, the experiment gives the mass of the earth in terms of that of the big sphere, and so its mean density. This experiment has often been repeated, but I do not think it is too much to say that no advance was made in exactness till we come to quite recent work.

By far the most remarkable recent study in gravitation is Prof. Boys' beautiful form of the Cavendish experiment, a research which stands out as a model in beauty of design and in exactness of execution (Fig. 2). But as Prof. Boys has described his ex. periment already in this theatre (Proc. R.I., xiv. Part ii. 1894 , p. 353), it is not necessary for me to more than refer to it. It is enough to say that he made the great discovery, obvious No. $6 \mathrm{c} 8$, roL. 627 
perhaps when made, that the sensitiveness of the apparatus is increased by reducing its dimensions. He therefore decreased the scale as far as was consistent with exact measurement of the parts of the apparatus, using a torsion rod, itself a mirror, only $2^{\prime \prime}$ long, gold balls, $m m$, only $1^{\prime \prime}$ in diameter, and attracting lead masses, M M, only $4 \frac{1}{4}^{\prime \prime}$ in diameter. The force to be measured was less than $1 / 5 \times 10^{6}$ grain.

The exactness of his work was increased by using as suspending wire one of his quartz threads. It would be difficult to over-estimate the service he has rendered in the measurement of small forces by the discovery of the remarkable properties of these threads.

One of the chief difficulties in the measurement of these small gravitational pulls is the disturbances which are brought about by the air currents, which blow to and fro and up and down inside the apparatus, producing irregular motions in the torsion rod. These, though much reduced, are not reduced in proportion to the diminution of the apparatus.

A very interesting repetition of the Cavendish experiment

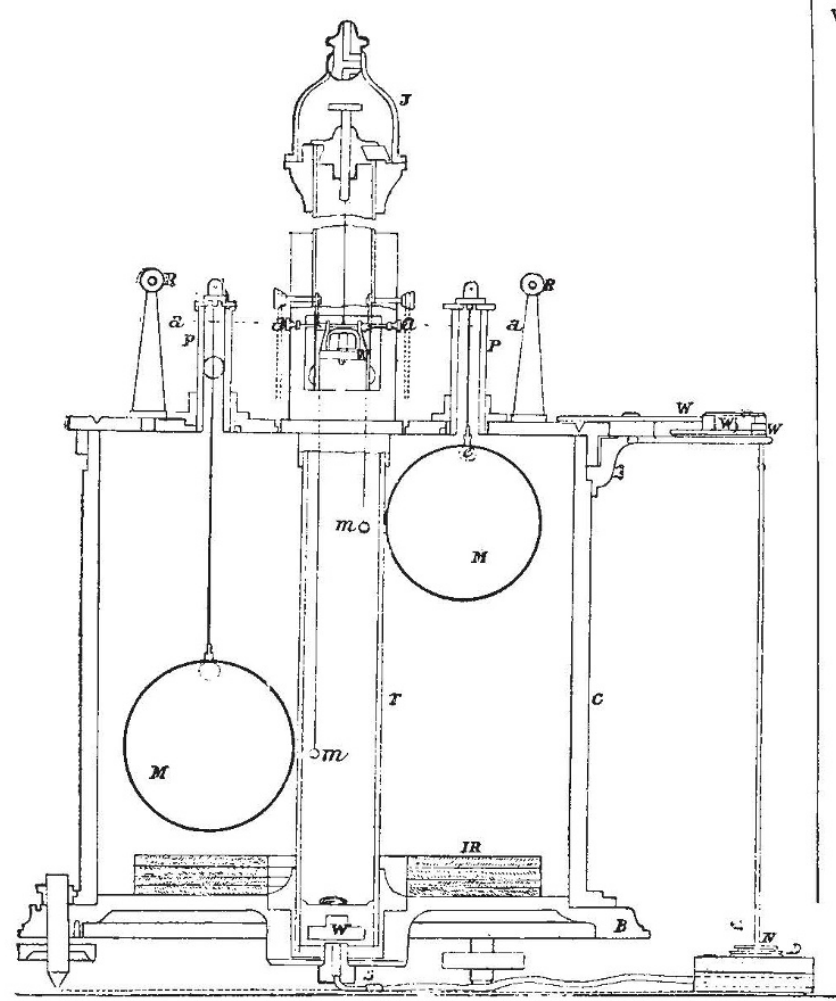

Fig. 2.-Boys' apparatus.

has lately been concluded by Dr. Braun (Denkschriften der Math. Wiss. Classe der Kais. Akad. der Wissenschaften Wien, lxiv. 1896) at Mariaschein, in Bohemia, in which he has sought to get rid of these disturbing air currents by suspending his torsion rod in a receiver which was nearly exhausted, the pressure being reduced to about $\frac{1}{2} 0$ of an atmosphere. The gales which have been the despair of other workers were thus reduced to such gentle breezes that their effect was hardly noticeable. His apparatus was nearly a mean proportional between that of Cavendish and Eoys, his torsion rod being about $9^{\prime \prime}$ long, the balls weighing 54 gms. - less than two ouncesand the attracting masses either 5 or 9 kgms. His work bears internal evidence of great care and accuracy, and he obtained almost exactly the same result as Prof. Boys.

Dr. Braun carried on his work far from the usual laboratory facilities, far from workshops, and he had to make much of his apparatus himself. His patience and persistence command our highest admiration.

I am glad to say that he is now repeating the experiment, using as suspension a quartz fibre supplied to hirn by Prof. Boys in place of the somewhat untrustworthy metal wire which he used in the work already published.

Prof. Boys has almost indignantly disclaimed that he was en. gaged on any such purely local experiment as the determination of the mean density of the earth. He was working for the Universe, seeking the value of $\mathrm{G}$, information which would be as useful on Mars or Jupiter or out in the stellar system as here on the earth. But perhaps we may this evening consent to be more parochial in our ideas, and express the results in terms of the mean density of the earth. In such terms, then, both Boys and Braun find that density 5.527 times the density of water, agreeing therefore to $\mathrm{I}$ in 5000 .

There is another mode of proceeding which may be regarded as the Cavendish experiment turned from a horizontal into a vertical plane, and in which the tursion balance is replaced by the common balance. This method occurred about the same time to the late Prof. V. Jolly and myself. The principle of my own experiment (Phil. Trans., I82, I891, A, p. 565) will be sufficiently indicated by Fig. 3. A big bullion balance with a 4 -foot beam had two lead spheres, A B, each about 5 olbs. in weight, hanging from the two ends in place of the usual scale

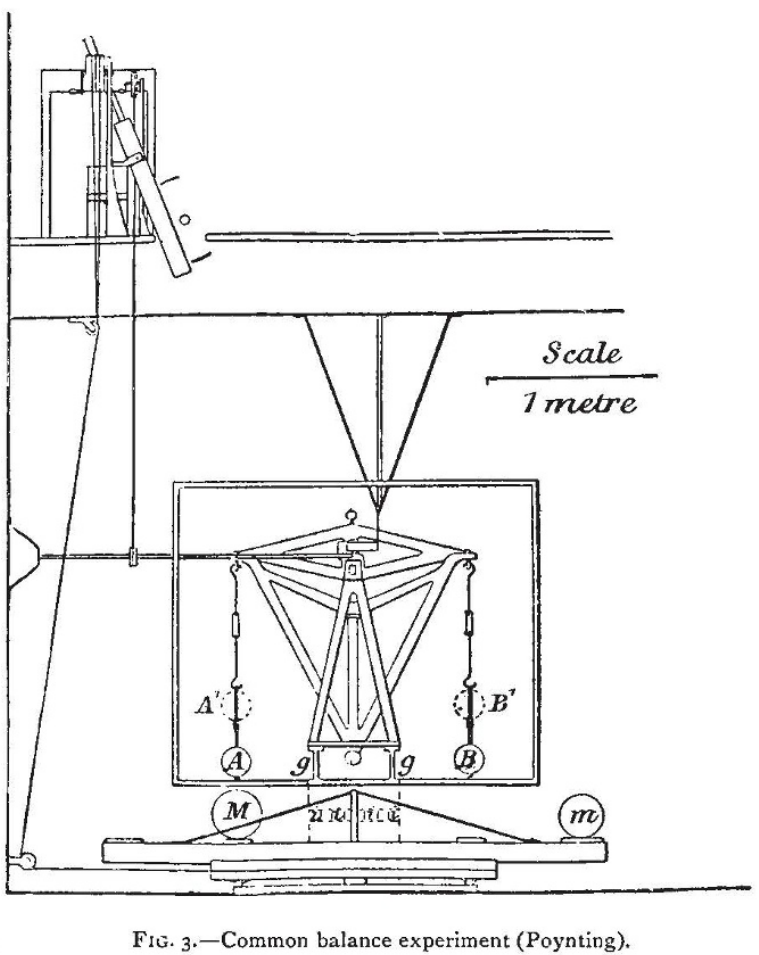

pans. A large lead sphere, $M, I^{\prime}$ in diameter and weighing about 350 Ibs., was brought first under one hanging weight, then under the other. The pull of the lead sphere acted first on one side alone and then on the other, so that the tilt of the balance beam when the sphere was moved round was due to twice the pull. By means of riders the tilt, and therefore the pull, was measured directly as so much increase in weight. This increase, when the sphere was brought directly under the hanging weight with $\mathbf{I}^{\prime}$ between the centres, was about $\frac{1}{8} \mathrm{mgm}$. in a total weight of $20 \mathrm{kgm}$., or about I in $100,000,000$. If, then, a sphere $\mathrm{I}$ foot away pulls with $\mathrm{I} / 10^{8}$ of the earth's pull, the earth being on the average $20,000,000$ feet away, it is easy to see that the earth's mass is calculable in terms of the mass of the sphere, and its density is at once deduced. The direct aim of this experiment, then, is not $\mathrm{G}$, but the mass of the earth.

It is not a little surprising that the balance could be made to indicate such a small increase in weight as I in 100 million. But not only did it indicate, it measured the increase, with variations usually well within I per cent. of the double attrac. tion, or to $\mathrm{I}$ in 5000 million of the whole weight, a change in weight which would occur merely if one of the spheres were moved $\frac{1}{\pi} \frac{1}{0}$ inch nearer the earth's centre. This accuracy is only 
attained by never lifting the knife edges and planes during an experiment, thus keeping the beam in the same state of strain throughout, and, further, by taking care that none of the mechanism for moving the weights or riders shall be attached in any way to the balance or its case, two conditions which are absolutely essential if we are to get the best results of which the balance is capable.

Quite recently another common balance experiment has been brought to a conclusion by Prof. Richarz and Dr. KrigarMenzel ("Anhang zu den Abhandlungen der Königl," Preuss. Akad. der Wissenschaften zu Berlin, I898) at Spandau, near Berlin. Their method may be gathered from Fig. 4. A balance of $23 \mathrm{~cm}$., say 9 -inch beam, was mounted above a huge lead pile about 2 metres cube, and weighing 100,000 kgm.

Two pans were supported from each end of the beam, one pan above, the other pan below the lead cube, the suspending wires of the lower pans going through narrow vertical tubular holes in the lead. Instead of moving the attracting mass, the attracted mass was moved. Masses of $1 \mathrm{kgm}$. each were put first, say, one in the upper right-hand pan, the other in the lower left-hand pan, when the pull of the lead block made the right hand heavier and the left hand lighter. Then the weights were changed to the lower right hand, and the upper left hand when the pulls of the lead pile were reversed. When we remember that in my experiment a lowering of the hanging

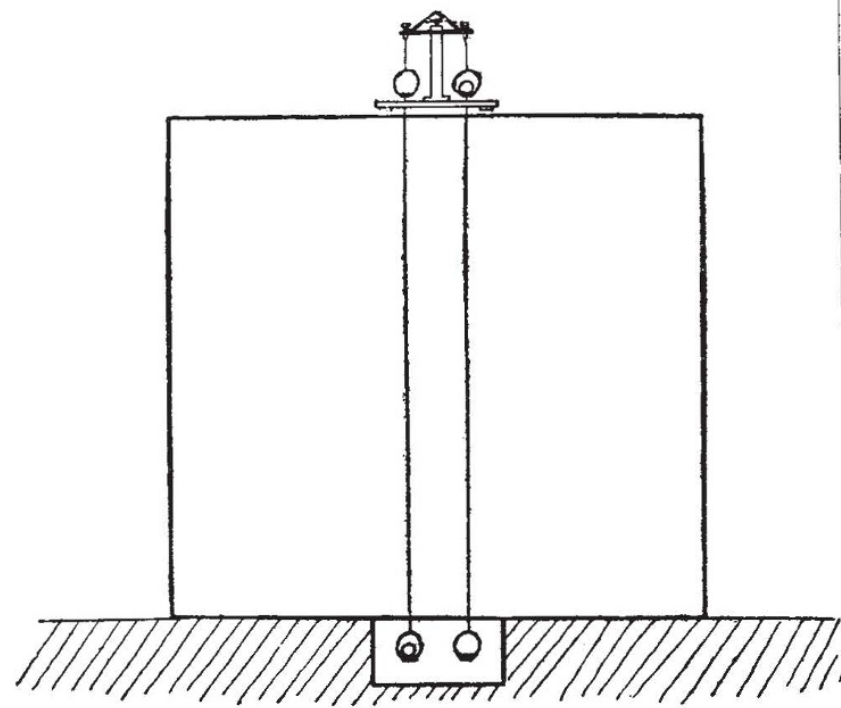

FIG. 4.-Common balance experiment (Richarz and Krigar-Menzel).

sphere by $\mathrm{I} \frac{1}{2}$ inches would give an effect as great as the pull I was measuring, it is evident that here the approach to and removal from the earth by over 2 metres would produce very considerable changes in weight, and, indeed, these changes masked the effect of the attraction of the lead. Preliminary experiments had, therefore, to be made before the lead pile was built up, to find the change in weight due to removal from upper to lower pan, and this change had to be allowed for. The quadruple attraction of the lead pile came out at I 3664 mgm., and the mean density of the earth at 5.505.

This agrees nearly with my own result of 5.49 , and it is a curious coincidence that the two most recent balance experiments agree very nearly at, say, $5{ }^{\circ} 5$; and the two most recent Cavendish experiments agree at, say, 5.53 . But I confess I think it is merely a coincidence. I have no doubt that the torsion experiment is the more exact, though probably an experiment on different lines was worth making. And I am quite content to accept the value of 5.527 as the standard value for the present.

And so the latest research has amply verified Newton's celebrated guess that "the quantity of the whole matter of the earth may be five or six times greater than if it consisted all of water."

I now turn to another line of gravitational research. When we compare gravitation with other known forces (and those No. 1608 , vOL. 62$]$ which have been most closely studied are electric and magnetic forces) we are at once led to inquire whether the lines of gravitative force are always straight lines radiating from or to the mass round which they centre, or whether, like electric and magnetic lines of force, they have a preference for some media and a distaste for others. We know, for example, that if a magnetic sphere of iron or cobalt or manganese is placed in a previously straight field, its permeability is greater than the air it replaces, and the lines of force crowd into it, as in Fig. 5.

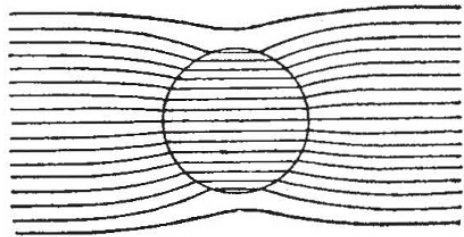

F1G. 5. - Paramagnetic sphere placed in a previously straight field.

The magnetic action is then stronger in the presence of the sphere near the ends of a diameter parallel to the original course of the lines of force, and the lines are deflected. If the sphere be diamagnetic, of water, or copper, or bismuth, the permeability being less than that of air, there is an opposite effect, as in Fig. 6, and the field is weakened at the end of a diameter parallel to the lines of force, and again the lines are deflected. Similarly, a dielectric body placed in an electric field gathers in the lines of force, and makes the field where the lines enter and leave stronger than it was before.

If we enclose a magnet in a hollow box of soft iron placed in a magnetic field, the lines of force are gathered into the iron and largely cleared away from the inside cavity, so that the magnet is screened from external action.

Now common experience might lead us at once to say that there is no very considerable effect of this kind with gravitation. The evidence of ordinary weighings may, perhaps, be rejected, inasmuch as both sides will be equally affected as the balance is commonly used. But a spring belance should show if there is any large effect when used in different positions above different media, or in different enclosures. And the ordinary balance is used in certain experiments in which one weight is suspended beneath the balance case, and surrounded, perhaps, by a metal case, or perhaps by a water bath. Yet no appreciable variation of weight on that account has yet been noted. Nor does the direction of the vertical change rapidly from place to place, as it would with varying permeability of the ground below. But perhaps the agreement of pendulum results, whatever the block on which the pendulum is placed, and whatever the case in which it is contained, gives the best evidence that there is no great gathering in, or opening out of the lines of the earth's force by different media.

Still, a direct experiment on the attraction between two masses with different media interposed was well worthy of trial, and such an experiment has lately been carried out in America

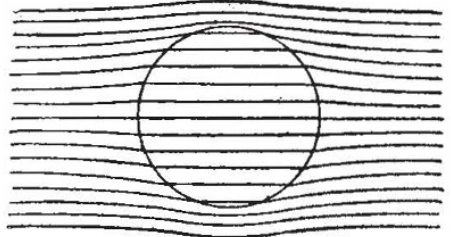

FIG. 6.-Diamagnetic sphere placed in a previously straight field.

by Messrs. Austin and Thwing (Physical Review, v. I897, p. 294). The effect to be looked for will be understood from Fig. 7. If a medium more permeable to gravitation is interposed between two bodies, the lines of force will move into it from each side, and the gravitative pull on a body, near the interposed medium on the side away from the attracting body, will be increased.

The apparatus they used was a modified kind of Boys' 
apparatus (Fig 8). Two small gold masses in the form of short vertical wires, each $4 \mathrm{gm}$. in weight, were arranged at different levels at the ends virtually of a torsion rod $8 \mathrm{~mm}$. long. The attracting masses $M_{1} M_{2}$ were lead, each about $I \mathrm{kgm}$. These were first in the positions shown by black lines in the figure, and were then moved into the positions shown by dotted lines. The attraction was measured first when merely the air and the case of the instrument intervened, and then when various slabs, each $3 \mathrm{~cm}$. thick, $10 \mathrm{~cm}$. wide and $29 \mathrm{~cm}$. high, were interposed. With screens of lead, zinc, mercury, water, alcohol or glycerine, the change in attraction was at the most about $I$ in 500, and this did not exceed the errors of experiment. That is, they found no evidence of a change in pull with change of medium. If such a change exists, it is not of the order of the change of electric pull with change of medium, but something far smaller. Perhaps it still remains just possible that there are variations of gravitation permeability comparable with the variations of magnetic permeability in media such as water and alcohol.

Yet another kind of effect might be suspected. In most crystalline substances the physical properties are different along different directions in a crystal. Triey expand differently, they conduct heat differently, and they transmit light at different speeds in different directions. We might, then, imagine that the lines of gravitative force spread out from, say, a crystal sphere unequally in different directions. Some years ago, Dr. Mackenzie (Physical Review, ii. 1895, p. 321) made an experiment in America in which he sought for direct evidence of such unequal distribution of the lines of force. He used a form of apparatus like that of Prof. Boys (Fig. 2), the attracting masses being calc spar spheres abuut 2 inches in

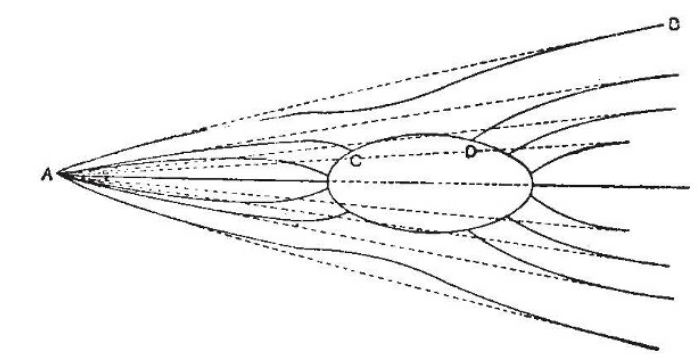

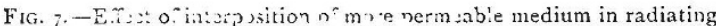
field of furce.

diameter. The attracted masses in one experiment were small lead spheres about $\frac{1}{2} \mathrm{gm}$. each, and he measured the attraction between the crystals and the lead when the axis of the crystals were set in various positions. But the variation in the attraction was merely of the order of error of experiment. In another experiment the attracted masses were small calc spar crystal cylinders weighing a little more than $\frac{1}{2} \mathrm{gm}$. each. But again there was no evidence of variation in the attraction with variation of axial direction.

Practically the same problem was attacked in a different way by Mr. Gray and myself (Phil. Trans., 192, 1899, A, p. 245). We tried to find whether a quartz crystal sphere had any directive action on another quartz crystal sphere close to it, whether they tended to set with their axes parallel or crossed.

It may easily be seen that this is the same problem by considering what must happen if there is any difference in the attraction between two such spheres when their axes are parallel and when they are crossed. Suppose, for example, that the attraction is always greater when their axes are parallel, and this seems a reasonable supposition, inasmuch as in straightforward crystallisation successive parts of the crystal are added to the existing crystal, all with their axes parallel. Begin, then, with tivo quartz crystal spheres near each other with their axes in the same plane, but perpendicular to each other. Remove one to a very great distance, doing work against their mutual attractions. Then, when it is quite out of range of appreciable action, turn it round till its axis is parallel to that of the fixed crystal. This absorbs no work if done slowly. Then let it return. The force on the return journey at every point is greater than the force on the outgoing journey, and more work will be got out than was put in. When the sphere is in its first position, turn it round till the axes are again at right angles.
Then work must be done on turning it through this right angle to supply the difference between the outgoing and incoming works. For if no work were done in the turning, we could go through cycle after cycle, always getting a balance of energy over, and this would, I think, imply either a cooling of the crystals or a diminution in their weight, neither supposition being admissible. We are led, then, to say that if the attraction with parallel axes exceeds that with crossed axes, there must be a directive action resisting the turn from the crossed to the parallel positions. And conversely, a directive action implies axial variation in gravitation.

The straightforward mode of testing the existence of this directive action would consist in hanging up one sphere by a wire or thread, and turning the other round into various positions, and observing whether the hanging sphere tended to twist out of position. But the action, if it exists, is so minute, and the disturbances due to air currents are so great, that it would be extremely difficult to observe its effect directly. It occurred to us that we might call in the aid of the principle of forced oscillations, by turning one sphere round and round at a constant rate, so that the couple would act first in one direction and then in the other, alternately, and so set the hanging sphere vibrating to and fro. The nearer the complete time of vibration of the applied couple to the natural time of vibration of the

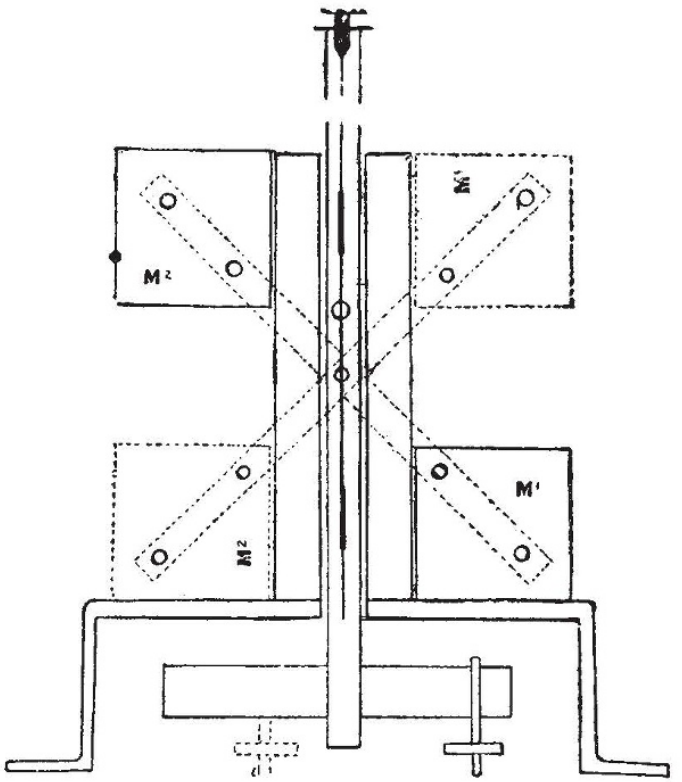

FIG. 8.-Experiment on gravitative permeability (Austin and Thwing).

hanging sphere, the greater would be the vibration set up. This is well illustrated by moving the point of suspension of a pendulum to and fro in gradually decreasing periods, when the swing gets longer and longer, till the period is that of the pendulum, and then decreases again. Or by the experiment of varying the length of a jar resounding to a given fork, when the sound suddenly swells out as the length becomes that which would naturally give the same note as the fork. Now, in looking for the couple between the crystals, there are two possible cases. The most likely is that in which the couple acts in one way while the turning sphere is moving from parallel to crossed, and in the opposite way during the next quarter turn from crossed to paraliel. That is, the couple vanishes four times during the revolution, and this we may term a quadrantal couple. But it is just possible that a quartz crystal has two ends like a magnet, and that like poles tend to like directions. Then the couple will vanish only twice in a revolution, and may be termed a semicircular couple. We looked for both, but it is enough now to consider the possibility of the quadrantal couple only.

Our mode of working will be seen from Fig. 9. The hanging sphere, $9 \mathrm{~cm}$. in diameter and $\mathrm{r} \mathrm{gm}$. in weight, was placed in a light aluminium wire cage with a mirror on it, and suspended by a long quartz fibre in a brass case with a window in it opposite the mirror, and surrounded by a double-walled tinfoiled wood 
case. The position of the sphere was read in the usual way by scale and telescope. The time of swing of this little sphere was I20 seconds.

A larger quartz sphere, $6.6 \mathrm{~cm}$. diameter and weighing 400 gms., was fixed at the lower end of an axis which could be

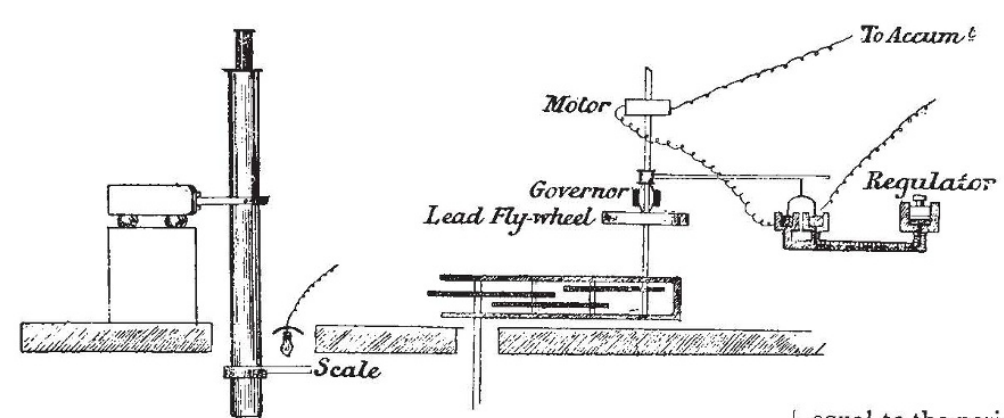

freer swing. The disturbances, which were mostly of an impulsive kind, continually set the hanging sphere into large vibration, and these might easily be taken as due to the revolving sphere. In fact, looking for the couple with exactly coincident periods would be something like trying to find if a fork set the air in a resonating jar vibrating when a brass band was playing all round it. It was necessary to make the couple period, then, a little different from the natural 120 second period, and, accordingly, we revolved the large sphere once in 230 seconds, when the supposed quadrantal couple would have a period of I I 5 seconds.

Figs. ro and II may help to show how this enabled us to eliminate the disturbances. Let the ordinates of the curves in Fig. Io represent vibrations set out to a horizontal time scale. The upper curve is a regular vibration of range \pm 3 , the lower a disturbance beginning with range \pm 10 . The first has period $\mathrm{I}$, the second period $\mathrm{I} \cdot 25$. Now cutting the curves into lengths

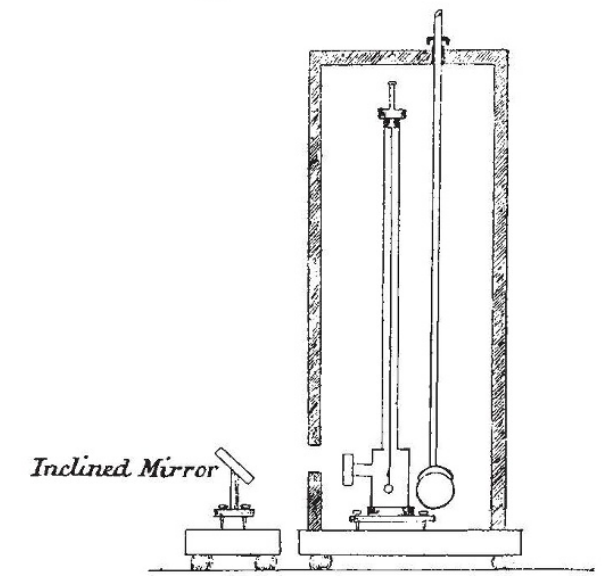

ime of vibration, and arranging the lengths one under the other as in Fig. II, it will be seen that the maxima and the minima of the regular vibration always fall at the same points, so that, taking 7 pericds and adding up the ordinates, we get 7 times the range, viz. $\pm 2 I$. But in the disturbance the maxima and minima fall at different points, and even with 7 periods only, the range is from +16 to -13 , or less than the range due to the addition of the much smaller regular vibration.

In our experiment, the couple, if it existed, would very soon establish its vibration, which would always be there and would go through all its values in I 5 seconds. An observer, watching the wheel at the top of the revolving axis, gave the time signals every $11 \cdot 5$ seconds, regulating the speed, if necessary, and an observer at the telescope gave the scale reading at every signal, that is, ro times during the period. The values were arranged in Io columns, each horizontal line giving the readings of a period. The experiment was carried on for about $2 \frac{1}{2}$ hours at a time, covering, say, 80 periods. On adding up the columns, the maxima and minima of the couple effect would always fall in the same
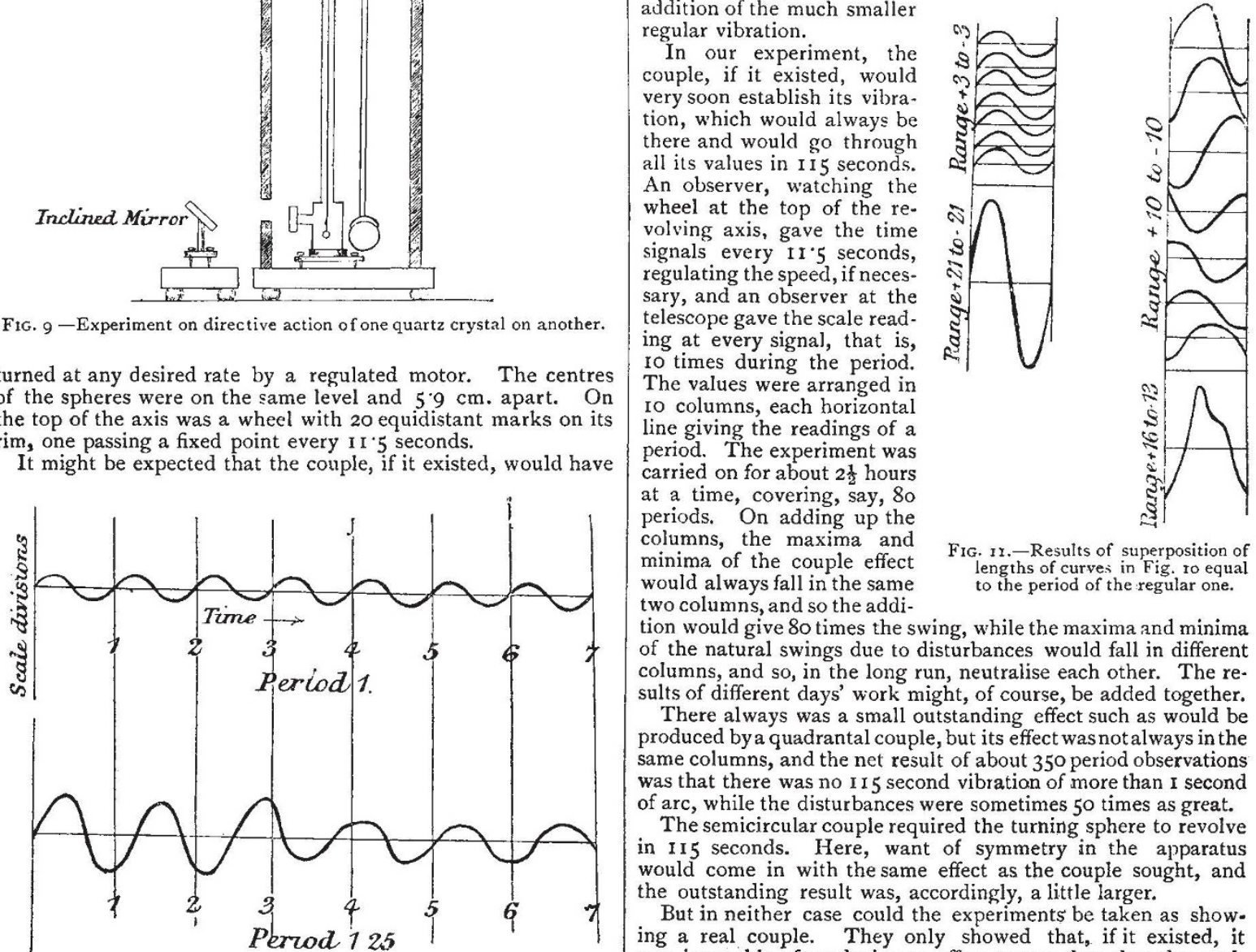

FIG. 10,-Upper curve a regular vibration. Lower curve a disturbance dying away.

the greatest effect if its period exactly coincided with the 120 second period of the hanging sphere-i.e. if the larger sphere revolved in 240 seconds. But in the conditions of the experiment the vibrations of the small sphere were very much damped, and the forced oscillations did not mount up as they would in a two columns, and so the addiwive 80 times the swing, while the maxima and minim of the natural swings due to disturbances would fall in different columns, and so, in the long run, neutralise each other. The results of different days' work might, of course, be added together.

There always was a small outstanding effect such as would be produced by a quadrantal couple, but its effect was not always in the same columns, and the net result of about 350 period observations was that there was no I I 5 second vibration of more than I second of arc, while the disturbances were sometimes 50 times as great.

The semicircular couple required the turning sphere to revolve in II seconds. Here, want of symmetry in the apparatus would come in with the same effect as the couple sought, and the outstanding result was, accordingly, a little larger.

But in neither case could the experiments be taken as show. ing a real couple. They only showed that, if it existed, it was incapable of producing an effect greater than that observed.

Perhaps the best way to put the result of our work is this: other. Then the couple is not greater than one which would take $5 \frac{1}{4}$ hours to turn it through that $45^{\circ}$ to the parallel position, and it would oscillate about that position in not less than 1,2 hours.

The semicircular couple is not greater than ane which would turn from crossed to parallel position in $4 \frac{1}{2}$ hours, and it would oscillate about that position is not less than 17 hours. Imagine the small sphere set with its axis at $45^{\circ}$ to that of the 
Or, if the gravitation is less in the crossed than in the parallel position, and in constant ratio, the difference is less than I in $I 6,000$ in the one case and less than $I$ in 2800 in the other.

We may compare with these numbers the difference of rate of travel of yellow light through a quartz crystal along the axis and perpendicular to it. That difference is of quite another order, being about $\mathrm{I}$ in $\mathbf{I} 7 \mathrm{O}$.

As to other possible qualities of gravitation, I shall only mention that quite indecisive experiments have been made to seek for an alteration of mass on chemical combination, ${ }^{1}$ and that at present there is no reason to suppose that temperature affects gravitation. Indeed, as to temperature effect, the agreement of weight methods and volume methods of measuring expansion with rise of temperature is good, as far as it goes, in showing that weight is independent of temperature.

So while the experiments to determine $\mathrm{G}$ are converging on the same value, the attempts to show that, under certain conditions, it may not be constant, have resulted so far in failure all along the line. No attack on gravitation has succeeded in showing that it is related to anything but the masses of the attracting and the attracted bodies. It appears to have no relation to physical or chemical condition of the acting masses or to the intervening medium.

Perhaps we have been led astray by false analogies in some of our questions. Some of the qualities we have sought and failed to find, qualities which characterise electric and magnetic forces, may be due to the polarity, the + and - , which we ascribe to poles and charges, and which have no counterpart in mass.

But this unlikeness, this independence of gravitation of any quality but mass, bars the way to any explanation of its nature.

The dependence of electric forces on the medium, one of Faraday's grand discoveries for ever associated with the Royal Institution, was the first step which led on to the electromagnetic theory of light now so splendidly illustrated by Hertz's electromagnetic waves. The quantitative laws of electrolysis, again due to Faraday, are leading, I believe, to the identification of electrification and chemical separation, to the identification of electric with chemical energy.

But gravitation still stands alone. The isolation which Faraday sought to break down is still complete. Yet the work I have been describing is not a failure. We at least know something in knowing what qualities gravitation does not possess, and when the time shall come for explanation all these laborious and, at first sight, useless experiments will take their place in the foundation on which that explanation will be built.

\section{SOCIETIES AND ACADEMIES.}

\section{PARIS.}

Academy of Sciences, August 13.-M. Maurice Lévy in the chair.-On the neogenic regions of Lower Egypt and the Isthmus of Suez, by MM. C. Depéret and R. Fourtau. Of the Miocene formation the following were recognised :-The Burdigalian, or first Mediterranean stratum, by the presence of Echinolampas amplus, Scutella Innesi, Lovenia, Cidaris avenionensis, Anphiope truncata and other fossils; the Vindobonian, or second Mediterranean stratum, by a blue lime containing Pecten cristato-costatus and numerous echinoderms. Of the Pliocene, in the neighbourhood of Cairo, are layers of yellowish sand containing Clypeaster aegyptiacus and other characteristic fossils.- The area of the basins of Russia in Asia, by M. J. de Schokalsky. The map is made upon the scale of I in $4,200,000$, and the area evaluated by means of a sheet of celluloid divided in square millimetres. The area found is $16,085,000$ sq. kilometres. - On a hypsometric map of European Russia, by M. J. de Schokalsky. The previous map of M. de Tillo was limited by the latitude $60^{\circ} \mathrm{N}$. ; the present map includes the whole of European Russia upon a scale of in $15,300,000$. - Observations of the Borelly comet, made at the Observatory of Algiers with the $31.8 \mathrm{~cm}$. equatorial, by M. F. Sy.-A new arrangement of apparatus serving to measure geodesic bases, by M. Alphonse Berget. Ruled plates of iron floating in a bath of mercury are used instead of the ordinary scales. The method has the advantage of securing without trouble the absolute horizontality of the rules; two consecutive rules are necessarily in the same horizontal plane, since their mercury baths are connected; there is no correction necessary for the flexure of the rules, and the temperature correction is much more certain. - Stereoscopic vision of curves traced by a phase apparatus, by M. Marc Dechevrens. - Properties of magnetic

${ }^{1}$ Landolt, Zeit. für Phys. Chem., xii. I, 1894. Sanford and Ray, Physical Reviere, v. 1897, p. 247.

NO. I 608, VOL. 62] deposits obtained in a magnetic field, by M. Ch. Maurain. Iron was deposited in a magnetic field either from a solution of ferrous and ammonium chlorides, or from a solution of ferrous sulphate in sodium pyrophosphate. It was found that the intensity of magnetisation of different layers of the deposit growing in a uniform field has the same value, and that the uniform mag. netisation acquired by a deposited strip increases with the strength of field in which the deposit is obtained. - The rôle of discontinuities in the propagation of explosive phenomena, by M. Paul Vieille. On the assumption of an adiabatic elasticity, together with continuity, the velocities of wave propagation found in certain cases are too great. It is necessary to assume that the phenomenon is discontinuous.-Action of hydrogen upon the sulphides of arsenic, by M. H. Pélabon. Details of experiments of the interaction of realgar and hydrogen in sealed tubes at $610^{\circ} \mathrm{C}$. The reaction is a reversible one, and the limit is affected by the introduction of an excess of arsenic.The properties of the blue oxide of molybdenum, by M. Marcel Guichard. The blue oxide is a molybdate, and could not be obtained free from water, two oxides of molybdenum only existing in the anhydrous state, $\mathrm{MoO}_{2}$ and $\mathrm{MoO}_{3}$. - On the colouring matter of Echinus esculentus, by M. Griffiths.-On the composition of the ashes of some medicinal plants, by $\mathrm{M}$. Griffiths.-On a cause of error in the examination of wines for salicylic acid, by M. J. Ferreira da Silva. The method of Petlet and Grobert will indicate the presence of salicylic acid in a pure wine that is really free from it. The official German method gives exact results. - On a variety of the anthrax bacillus; a short asporogenic form, Bacillus anthracis brevigemmans, by M. C. Phisalix. In the organism of the $\operatorname{dog}$ the $B$. anthracis undergoes important modifications, becoming shorter with a rapid and complete segmentation. It is still uncertain whether this should be regarded as a variety or a new species.-Antihepatic serum, by M. C. Delezenne.-Application to man of the regeneration of confined air by means of sodium peroxide, by MM. A. Desgrez and V. Balthazard. The apparatus described weighs twelve kilograms, and by its means a man can penetrate easily into an irrespirable atmosphere.

\section{CONTENTS.}

A Museum Catalogue 385

A Text-book of Mammals. By R. L. . . . . . . . 386 Good and Bad Air. By J. B. C. . . . . . . . 387 Our Book Shelf:-

Le Dantec : " Lamarckiens et Darwiniens ; Discussion de quelques Théories sur la Formation des Espèces."-F. A. D.

"Helen Keller : Souvenir"

Binet : "The Psychology of Reasoning": . . 388

"Electric Batteries: How to Make and Use Them" 388

Letters to the Editor:-

Snowdrifts on Ingleborough in July.-Prof. T. McKenny Hughes, F. R.S. . . . . . . The Total Eclipse of the Sun of May i7-18, I9or. -J.J. A. Muller

The Reform of Mathematical Teaching...David Mair 38 Functions of an Organ of the Larva of the Puss Moth. -Arthur S. Thorn

Dark Images of Photographed Lightning Discharges. -J. B. Hannay

The Lavoisier Monument. (Illustrated.) : : . 389

Nile Floods and Monsoon Rains . . . . . . . . 39I The Forthcoming Meeting of the British Association at Bradford. By Ramsden Bacchus . . . . . . 392

Notes

Our Astronomical Column:-

Velocities of Meteors

Standards for Faint Stellar Magnitudes

The Total Solar Eclipse, May 28,1900

Report of the Cape Observatory. ..... 398

Rousdon Observatory (Devon) ....... 398

Independent Day Numbers for 1902 . $\cdot . \cdot 398$

The August Perseids of 1900 . By W. F. Denning 398

What Pressure is dangerous on Electric Railways with Overhead Trolley Wires. By William Rung 399 Sea-coast Destruction and Littoral Drift. By W. H. Wheeler

Recent Studies in Gravitation. (Illustrated.) By Prof. John H. Poynting, F.R.S. . . . . . 403 Societies and Academies ......... 408 (1) 392 399 98 \begin{tabular}{l}
398 \\
98 \\
\hline 98
\end{tabular} 8 98
98 